\title{
Systematic Early Urinary Catheter Removal Integrated in the Full Enhanced Recovery After Surgery (ERAS) Protocol After Laparoscopic Mid to Lower Rectal Cancer Excision: A Feasibility Study
}

\author{
Hélène Meillat ${ }^{1}$, Cloé Magallon², Clément Brun², Cécile de Chaisemartin, Laurence Moureau-Zabotto ${ }^{3}$, \\ Julien Bonnet ${ }^{1}$, Marion Faucher ${ }^{2}$, Bernard Lelong ${ }^{1}$ \\ ${ }^{1}$ Department of Digestive Surgical Oncology, Institut Paoli Calmettes, Marseille; ${ }^{2}$ Polyvalent Intensive Care Unit, Department of Anesthesiology \\ and Critical Care, Institut Paoli Calmettes, Marseille; ${ }^{3}$ Department of Radiation Therapy, Institut Paoli Calmettes, Marseille, France
}

Purpose: Enhanced Recovery After Surgery (ERAS) programs advocate early urinary catheter removal after rectal cancer surgery; however, the optimal duration remains unclear. This study assessed the feasibility of the early urinary catheter removal protocol after rectal cancer surgery within an ERAS pathway and identified predictive factors for failure of this strategy.

Methods: Between March 2017 and October 2018, all unselected and consecutive patients who underwent rectal cancer resection and benefited from our ERAS program were included. Urinary complications (infection and retention) were prospectively recorded. Success was defined as catheter removal on postoperative day (POD) 3 without urinary complications. Results: Of 135 patients (male, 63.7\%; neoadjuvant chemoradiation, $57.0 \%$; urology history, $17.8 \%$ ), 120 had early urinary catheter removal with no complications (success rate, 88.9\%), 8 did not have urinary catheter removal on POD 3 due to clinical judgment or prescription error, 5 experienced a urinary tract infection, and 2 had acute urinary retention. Obesity (odds ratio $[\mathrm{OR}], 0.16 ; \mathrm{P}=0.003)$, American Society of Anesthesiologists physical status classification $>$ II $(\mathrm{OR}, 0.28 ; \mathrm{P}=$ $0.048)$, antiaggregant platelet medication $(\mathrm{OR}, 0.12 ; \mathrm{P}<0.001)$, absence of anastomosis $(\mathrm{OR}, 0.1 ; \mathrm{P}=0.003)$, and prolonged operative time $(\mathrm{OR}, 0.21 ; \mathrm{P}=0.020)$ were predictive factors for failure. Conversely, optimal compliance with the ERAS program $(\mathrm{OR}, 7.68 ; \mathrm{P}<0.001)$, postoperative nonsteroidal anti-inflammatory drug use $(\mathrm{OR}, 21.71 ; \mathrm{P}<0.001)$, and balanced intravenous fluid therapy $(\mathrm{OR}, 7.87 ; \mathrm{P}=0.001)$ were associated with increased strategy success.

Conclusion: Withdrawal of the urinary catheter on POD 3 was successfully achieved after laparoscopic rectal resection and can be safely implemented in the ERAS program.

Keywords: Colorectal surgery; Surgical oncology; Enhanced Recovery After Surgery, Urinary retention

\section{INTRODUCTION}

Despite autonomic nerve preservation, perioperative urinary

Received: Mar 19, 2020 - Revised: May 11, 2020 - Accepted: May 22, 2020 Correspondence to: Hélène Meillat, M.D.

Department of Digestive Surgical Oncology, Institut Paoli Calmettes, 232 Boulevard Sainte Marguerite, Marseille 13009, France

Tel: +33-491223660, Fax: +33-491223550

E-mail: meillath@ipc.unicancer.fr

ORCID: https://orcid.org/0000-0003-4548-6481

(C) 2021 The Korean Society of Coloproctology

This is an open-access article distributed under the terms of the Creative Commons Attribution NonCommercial License (https://creativecommons.org/licenses/by-nc/4.0) which permits unrestricted noncommercial use, distribution, and reproduction in any medium, provided the original work is properly cited. catheterization is required after rectal cancer surgery to provide guidance for volume resuscitation and hemodynamic stability maintenance, ensure bladder decompression, and avoid postoperative acute urinary retention (AUR). However, in this era of care standardization and Enhanced Recovery After Surgery (ERAS) programs for colorectal surgery, the management of urinary catheterization requires adjustment. Adverse events such as urinary tract infection $[1,2]$ or restriction of patient mobilization $[3,4]$ have led to the recommendation that the catheter should be removed early after colorectal resection [5]. ERAS guidelines recommend urinary catheter removal on the postoperative day (POD) 1 whenever possible for selected patients. However, they also recommend the insertion of a suprapubic catheter if postop- 
erative drainage exceeding 4 days is planned. Therefore, surgeons must prejudge the risk of acute urine retention. Risk factors for urinary retention are numerous and controversial. Some of these factors are not modifiable, including age, male sex, and comorbidities $[6,7]$, and other factors are modifiable and might result from changes in anesthetic and surgical practices associated with the ERAS program. To our knowledge, there are insufficient data concerning the optimal duration of urinary drainage after rectal resection within an optimal ERAS program. This study aimed to assess the safety of a standardized protocol involving early urinary catheter removal after rectal cancer surgery within an ERAS program and to identify predictive factors for failure of this strategy.

\section{METHODS}

In March 2017, a standardized ERAS program for elective rectal resection was implemented at the Paoli Calmettes Institute and applied systematically. Therefore, we prospectively evaluated all consecutive patients undergoing rectal cancer resection between March 2017 and April 2018. The inclusion criteria were as follows: patients older than 18 years and elective rectal resection for histopathologically confirmed adenocarcinoma requiring dissection of the infraperitoneal portion of the rectum. Patients who needed emergency surgery and those requiring multivisceral resection or an abdominoperineal resection were excluded. During the observation period, 140 patients underwent rectal resection by a dedicated colorectal surgical team and 5 patients were excluded due to prolonged urinary catheterization implemented for the following objective reasons: intraoperative urinary tract invasion, 2; surgical complication before POD 3 requiring reintervention, 2 (pelvic abscess, 1; anastomotic hemorrhage, 1); and guidance for volume resuscitation after rhabdomyolysis with chronic renal failure, 1 . Therefore, our study population comprised of 135 patients (Fig. 1).

This study was approved by the Institutional Review Board and Ethics Committee of Institut Paoli Calmettes, Marseille (NCT02869503) and informed consent was obtained from all the subjects. It was performed in accordance with the 1964 Helsinki Declaration and its later amendments or comparable ethical standards.

\section{Preoperative management}

Systematic pretherapeutic evaluations included chest, abdominal, and pelvic computed tomography scans, serum carcinoembryonic antigen measurements, endorectal ultrasound examinations, and rectal magnetic resonance imaging (MRI).

At our institution, preoperative treatment is indicated for $\mathrm{T} 3$ and/or $\mathrm{N}+$ mid and low rectal adenocarcinoma and for T2 lowlying tumors close to the sphincter. Standard chemoradiation (CRT) comprised a total dose of $50 \mathrm{~Gy}$, with a daily dose of $2 \mathrm{~Gy}$ combined with capecitabin.

Patients with a predictable threatened circumferential resection

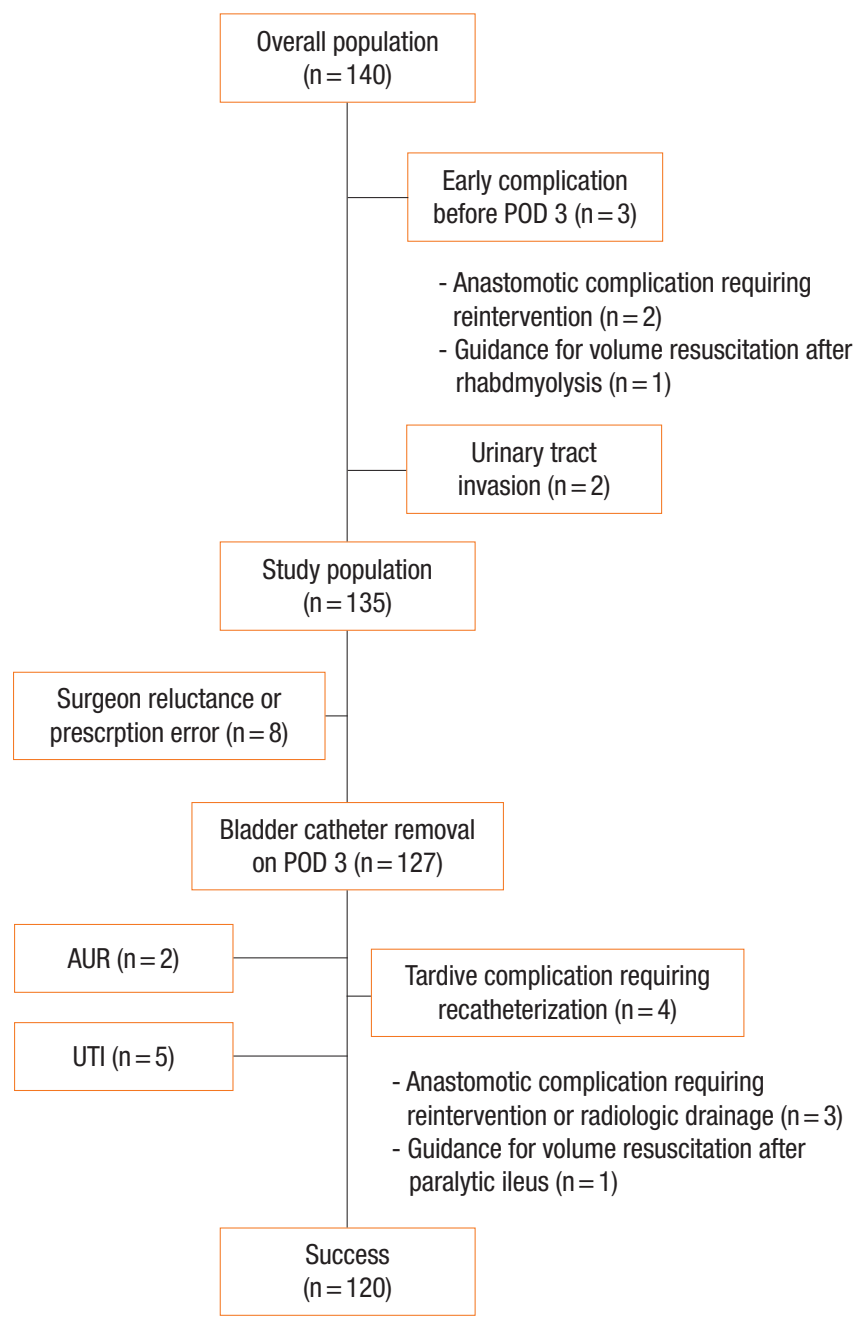

Fig. 1. Flowchart of patient selection. POD, postoperative day; AUR, acute urinary retention; UTI, urinary tract infection.

margin on MRI underwent an intensified preoperative protocol that comprised induction chemotherapy (FOLFIRINOX [fluorouracil, leucovorin, irinotecan, and oxaliplatin], 4 cycles) followed by standard CRT.

Surgery was usually performed 8 to 12 weeks after completing chemoradiotherapy. Patients underwent systematic bowel mechanical preparation without preoperative oral antibiotics.

\section{Enhanced Recovery After Surgery program and surgery}

A standardized patient clinical pathway was developed in accordance with the ERAS recommendations and published guidelines [5] that define more than 20 perioperative standard care elements (Table 1). In the operating room, the urinary catheter was inserted after induction of general anesthesia under aseptic conditions (no bacteriological examination was routinely performed). No epidural analgesia was administered at our institution, but continuous perfusion of lidocaine was maintained until the last hour of 
Table 1. Patients characteristics and operative data

\begin{tabular}{|c|c|c|c|}
\hline Variable & $\begin{array}{l}\text { Success } \\
(n=120)\end{array}$ & $\begin{array}{l}\text { Failure } \\
(n=15)\end{array}$ & P-value ${ }^{a}$ \\
\hline Age (yr) & $64 \pm 11.8$ & $69.3 \pm 9.23$ & 0.140 \\
\hline Sex, male:female & $74: 46$ & $12: 3$ & 0.270 \\
\hline Body mass index $\left(\mathrm{kg} / \mathrm{m}^{2}\right)$ & $24.6 \pm 4.05$ & $28.7 \pm 5.91$ & $<0.010$ \\
\hline Malnutrition & $14(11.7)$ & $2(13.3)$ & 0.690 \\
\hline \multicolumn{4}{|l|}{ ASA PS classification } \\
\hline I & 27 (22.5) & $1(6.7)$ & 0.080 \\
\hline$\|$ & $78(65.0)$ & $9(60.0)$ & \\
\hline III & $15(12.5)$ & $5(33.3)$ & \\
\hline \multicolumn{4}{|l|}{ Comorbidity } \\
\hline Respiratory & $15(12.5)$ & $3(20.0)$ & 0.420 \\
\hline Cardiovascular & $43(35.8)$ & $9(60.0)$ & \\
\hline Diabetes & $12(10.0)$ & $3(20.0)$ & 0.370 \\
\hline Tobacco & $53(44.2)$ & $8(53.3)$ & 0.690 \\
\hline Previous abdominal surgery & $46(38.3)$ & $6(40.0)$ & $>0.999$ \\
\hline Urology history & $20(16.7)$ & $4(26.7)$ & 0.310 \\
\hline Platelet antiaggregant & $14(11.7)$ & $8(53.3)$ & $<0.001$ \\
\hline Prior radiotherapy & $66(55.0)$ & $12(80.0)$ & 0.120 \\
\hline Metastatic disease & $16(13.3)$ & $1(6.7)$ & 0.690 \\
\hline \multicolumn{4}{|l|}{ Surgical approach } \\
\hline Laparoscopic & $82(68.3)$ & $10(66.7)$ & $>0.999$ \\
\hline Robotic & $38(31.7)$ & $5(33.3)$ & \\
\hline \multicolumn{4}{|l|}{ Surgical procedure } \\
\hline PME & 43 (35.8) & $0(0)$ & $<0.010$ \\
\hline TME & 77 (64.2) & $15(100)$ & \\
\hline Anastomosis & $114(95.0)$ & $10(66.7)$ & $<0.010$ \\
\hline Ileostomy & 85 (70.8) & $10(66.7)$ & 0.060 \\
\hline Operative time (min) & $385 \pm 88.58$ & $328 \pm 68.89$ & $<0.010$ \\
\hline Conversion to open & $3(2.5)$ & $1(6.7)$ & 0.380 \\
\hline \multicolumn{4}{|l|}{ Tumor location } \\
\hline Anterior & 45 (37.5) & $10(66.7)$ & 0.060 \\
\hline Posterior & $75(62.5)$ & $5(33.3)$ & \\
\hline \multicolumn{4}{|l|}{ Tumor classification } \\
\hline рT0-2 & $60(50.0)$ & $10(66.7)$ & 0.280 \\
\hline pT3-4 & $60(50.0)$ & $5(33.3)$ & \\
\hline Length of hospital stay (day) & $8.17 \pm 4.22$ & $12.8 \pm 3.85$ & $<0.010$ \\
\hline
\end{tabular}

Values are presented as mean \pm standard deviation or number (\%).

ASA, American Society of Anesthesiologists; PS, physical status; PME, partial mesorectal excision; TME, total mesorectal excision; $\mathrm{pT}$, primary tumor.

${ }^{a}$ Chi-square, Fisher, or Wilcoxon test.

the surgical procedure in association with locoregional analgesia (transversus abdominis plane block or trocar site local anesthetic infiltration). All rectal surgery procedures were performed using a minimally invasive approach. High tie ligation of the inferior mesenteric artery was standardized within our team, and care was taken to preserve the superior hypogastric plexus. The bilateral hypogastric nerves were identified at the sacral promontory, and dissection was performed along the avascular plane. Systematic identification and preservation of the lateral pelvic plexus were also achieved. A temporary ileostomy was performed for restorative procedures; it was performed systematically in the case of total mesorectal excision (TME) and selectively for partial mesorectal excision (PME) cases.

Postoperative pain was managed with a combination of paracetamol, opioids, and nonsteroidal anti-inflammatory drugs (NSAID) over the course of 48 hours. Oral morphine was reserved for patients with persistent pain despite the protocol.

The urinary catheter was removed after at least 2 days of treatment with an alpha-blocker. This treatment was introduced as soon as possible after surgery. As it is not always possible to administer this treatment on the same evening after the procedure, we formalized urinary catheter removal within 72 hours after the surgical procedure (POD 3). In parallel, our local ERAS protocol involved the planned removal of intravenous fluid infusion and pelvic drainage on POD 2 to allow early free ambulation. Except for patients treated with long-term alpha-blockers, the treatment was suspended 2 days after successful urinary catheter removal.

\section{Outcomes}

The primary outcome measure was the feasibility of a protocol for early urinary catheter removal after rectal cancer surgery within an ERAS program. Success was defined as catheter removal within 72 hours after surgery (POD 3) without urinary complications such as urinary tract infection (UTI) and AUR. UTI was defined as clinical infection confirmed by positive urine culture results. AUR was defined as the absence of spontaneous micturition 12 hours after catheter removal. During the first 2 days after catheter removal, 24-hour diuresis monitoring was performed. Ultrasound evaluation of the postvoid residual was performed only in cases of symptoms suggesting overflow incontinence (loss of bladder control, spontaneous lower abdominal pain or on clinical examination, weak urine stream) and low 24-hour diuresis. The urinary catheter was reinserted when the postvoid residual was more than $100 \mathrm{~mL}$. Postoperative catheter reinsertion for fluid guidance or reoperation was not considered a treatment failure for the purposes of the study. Risk factors for failure were identified as demographic, surgery-related, and perioperative care items.

\section{Data collection and follow-up}

Preoperative, perioperative, and postoperative data until POD 90 were recorded routinely in a prospective database. Demographic information included age, sex, body mass index (BMI), American Society of Anesthesiologists (ASA) physical status (PS) classification, comorbidities, and previous abdominal surgery. Surgical information included the main procedure (PME or TME), surgical 
approach (laparoscopic or robotic surgery), duration, and combined procedures.

All perioperative care items of our program were prospectively recorded. Overall compliance with 20 items was assessed and expressed as a percentage. Good compliance was defined as a score of $\geq 80 \%$ per criteria and/or per patient.

Patients underwent a follow-up outpatient evaluation 7 to 10 days after discharge. Any hospitalization of the patient within 30 days after surgery and after being discharged home was considered as readmission.

\section{Statistical analysis}

Descriptive data were reported as the mean and standard deviation for quantitative variables and percentages for categorical variables. To test the effects of potential factors on the risk of failure, means were compared using the Student $\mathrm{t}$-test for normally distributed data or the Mann-Whitney U-test for nonnormally distributed data. Proportions were compared using the chi-square test or Fisher exact test, as appropriate. Odds ratios were presented with their $95 \%$ confidence intervals. A two-sided significance level of $<0.05$ was considered statistically significant. Analyses were conducted with R 3.5.1 (R Foundation for Statistical Computing, Vienna, Austria).

\section{RESULTS}

Patient characteristics and operative data are summarized in Table $1 ; 24$ patients $(17.8 \%)$ had a history of urologic conditions (17 with benign prostatic hyperplasia, 5 with prostate cancer treated by radiotherapy and/or radical surgery, and 2 with surgery for relapse). Seventy-seven patients (57.0\%) underwent preoperative CRT. Forty-three patients (31.9\%) underwent PME and 94 (69.6\%) underwent TME. Overall, compliance with the ERAS program was $95.5 \%$ (Table 2).

\section{Urinary catheter management and outcomes}

For 127 patients (94.1\%), the urinary catheter was removed according to the protocol within 72 hours postoperatively, i.e. on POD $2(n=84)$ or on POD $3(n=43)$. Among them, the urinary catheter was removed on POD 2 in 84 patients (62.2\%). The protocol was successful for 120 patients (88.9\%) and protocol failure was noted in 15 patients; 8 patients did not have urinary catheter removal on POD 3 due to clinical judgment or prescription error, 5 patients experienced a UTI, and 2 patients had AUR.

Recatheterization was required for 4 patients (3.0\%) without urinary complications due to the need for fluid guidance $(n=1)$ or reoperation $(\mathrm{n}=3)$; those patients were not considered as a protocol failure because they urinated normally for at least 48 hours after the first catheter removal. The main causes of protocol failure are summarized in Fig. 1.

The univariate analysis (Table 3) identified obesity, ASA PS classification > II, antiaggregant platelets, no anastomosis, and pro- longed operative time ( $>326$ minutes) as protocol failure factors. Optimal compliance with the ERAS program (all 20 items), postoperative NSAID use, and balanced intravenous fluid therapy were associated with an increased success rate.

\section{DISCUSSION}

Our prospective study showed that early removal of the urinary catheter on POD 3 after rectal resection for cancer is safe and reproducible within an optimal ERAS program. This is the first study to establish a link between optimal compliance with an ERAS program and urinary outcomes after rectal cancer surgery.

Historically, postoperative urinary catheterization for several days after rectal cancer surgery has been routinely performed because of concerns regarding the AUR risk if the superior hypogastric nerves and/or lateral pelvic plexus were damaged during dissection. Additionally, patients may require prolonged urinary catheterizations because of anesthetic factors such as hemodynamic monitoring, risk of AUR in the case of epidurals, or overuse of opioids. However, prolonged catheterization is an invasive procedure with potential related morbidity, including UTI, urethral trauma, prostatitis, and patient discomfort [6]. Therefore, some authors have suggested suprapubic catheter placement as an alternative, which has been associated with reduced UTI rates, recatheterization rates, and pain compared with urethral placement [7].

However, 3 changes led us to question this policy. First, the development of an optimal TME technique and anatomic knowledge of the pelvis minimize any AUR resulting from trauma [810]. Second, minimally invasive approaches (laparoscopic and robotic surgery) showed a significant reduction in postoperative morbidity. However, some studies found that laparoscopic surgery was an independent risk factor for AUR after rectal cancer surgery $[11,12]$. They suggest that the increase in intraperitoneal pressure during pneumoperitoneum disturbs renal function and renal blood flow, artificially reducing intraoperative urine output. However, it recovers after surgery and may cause greater urination during the early postoperative period in the case of excessive perioperative fluid and may lead to overdistension of the bladder, decreased sensation and bladder contractility, and urinary retention. Balanced fluid administration ( $3 \mathrm{~mL} / \mathrm{kg} /$ hour) and also lowpressure laparoscopy (at 8 or $10 \mathrm{mmHg}$ ) are key components of our program for preventing AUR. Third, the ERAS program implementation changed the perioperative management of patients; in particular, we have seen a decrease in opioid use and questioning of the usefulness of suprapubic catheter placement because it caused patients discomfort and pain and delayed ambulation [13]. ERAS guidelines promote early urinary catheter removal after colorectal surgery, but there are insufficient data concerning the optimal duration of urinary drainage after rectal resection. Reports of AUR after colorectal surgery vary widely in the literature, between $2 \%$ and $50 \%[3,13]$, and 2 randomized studies $[3,14]$ 


\section{Coloproctology Helene Meillat, et al.}

Table 2. ERAS protocol and compliance

\begin{tabular}{|c|c|c|c|c|c|}
\hline ERAS protocol & Detailed measure & Overall $(n=135)$ & Success $(n=120)$ & Failure $(n=15)$ & P-value ${ }^{a}$ \\
\hline 1. Specific information & Information about the fast track program & $132(97.8)$ & $117(97.5)$ & $15(100)$ & $>0.999$ \\
\hline 2. Immunonutrition & & $135(100)$ & - & - & - \\
\hline 3. Limited fast & $\begin{array}{l}\text { Last meal } 6 \text { hr before surgery, clear fluids until } \\
2 \text { hr before surgery }\end{array}$ & $134(99.3)$ & $120(100)$ & $14(93.3)$ & 0.11 \\
\hline 4. Carbohydrate loading & $\begin{array}{l}200 \mathrm{~mL} \text { the evening and } 200 \mathrm{~mL} 2 \mathrm{hr} \text { before } \\
\text { surgery }\end{array}$ & $125(92.6)$ & $113(94.2)$ & $12(80.0)$ & 0.08 \\
\hline 5. No long-acting sedation & If necessary & $120(88.9)$ & $106(88.3)$ & 14 (93.3) & $>0.999$ \\
\hline 6. Antibiotic prophylaxis & & $135(100)$ & - & - & - \\
\hline 7. IV Lidocaine & $\begin{array}{l}\text { Continuous perfusion of lidocaine }(1 \mathrm{mg} / \mathrm{kg} / \mathrm{hr}) \\
\text { during surgery, maintained until the last hour of } \\
\text { the surgical procedure }\end{array}$ & $135(100)$ & - & - & - \\
\hline 8. Laparoscopic approach & & $134(99.3)$ & $119(99.2)$ & $15(100)$ & $>0.999$ \\
\hline 9. Zero fluid balanced & $\begin{array}{l}\text { Optimized hemodynamic monitoring with } \\
\text { goal-directed intraoperative fluid infusion regime } \\
(<3 \mathrm{~mL} / \mathrm{kg} / \mathrm{hr})\end{array}$ & $119(88)$ & $108(90.0)$ & 8 (53.3) & $<0.010$ \\
\hline 10. Corticosteroid & & $135(100)$ & - & - & - \\
\hline 11. PONV prevention & $\begin{array}{l}\text { Prophylactic use of metoclopramide ondansetron } \\
(4 \mathrm{mg}) \text { and/or }\end{array}$ & $107(79.3)$ & $104(86.7)$ & $11(73.3)$ & 0.23 \\
\hline 12. Normothermia & Forced body heating (Bair hugger system) & $135(100)$ & - & - & - \\
\hline 13. Avoidance of nasogastric tube & Removal of nasogastric tube before extubating & $134(99.3)$ & $119(99.2)$ & $15(100)$ & $>0.999$ \\
\hline $\begin{array}{l}\text { 14. Preventive opioid-sparing multimodal } \\
\text { analgesia including locoregional analgesia }\end{array}$ & $\begin{array}{l}\text { Infiltration of surgical wounds with lidocaine No } \\
\text { epidural analgesia }\end{array}$ & $117(86.7)$ & $103(85.8)$ & $12(80.0)$ & 0.47 \\
\hline 15. Postoperative NSAl agents & Ketoprofen 200 mg/day during 24-48 hr & $121(89.6)$ & $114(95.0)$ & $7(46.7)$ & $<0.001$ \\
\hline 16. Free diet on POD 1, early on POD 3 & Liquid diet on POD 0 Normal diet on POD 1 & $126(93.3)$ & $114(95.0)$ & $12(80.0)$ & 0.06 \\
\hline 17. Gum & & $132(97.8)$ & $118(98.3)$ & $13(86.7)$ & 0.06 \\
\hline 18. Early mobilization out of bed on POD 0 & Out of bed $1 \mathrm{hr}$ on POD 0 and $4 \mathrm{hr}$ on POD 1 & $105(77.8)$ & $96(80.0)$ & $7(46.7)$ & $<0.010$ \\
\hline $\begin{array}{l}\text { 19. Early termination of IV fluid infusion on } \\
\text { POD } 3\end{array}$ & $\begin{array}{l}\text { Limited intake }(<1,500 \mathrm{~mL} / \text { day) on POD } 1 \mathrm{IV} \\
\text { catheter removal on POD } 2\end{array}$ & $116(85.9)$ & $105(87.5)$ & $11(73.3)$ & 0.23 \\
\hline 20. TED prophylaxis & $\begin{array}{l}\text { Compression stockings before surgery Prophylactic } \\
\text { anticoagulation }\end{array}$ & $135(100)$ & - & - & - \\
\hline Compliance with ERAS protocol (\%) & & $19.2(96.0)$ & $19.3(96.5)$ & $17.8(89.0)$ & $<0.001$ \\
\hline
\end{tabular}

Values are presented as number (\%).

ERAS, Enhanced Recovery After Surgery; IV, intravenous; PONV, postoperative nausea/vomiting; NSAI, nonsteroidal anti-inflammatory; POD, postoperative day; TED, thromboembolic disease.

${ }^{a}$ Chi-square, Fisher, or Wilcoxon test.

that examined the duration of urinary catheterization following rectal resection without any ERAS program supported earlier catheter removal, but without a proposed duration. Low rectal cancer and TME were associated with AUR in both studies, with a high UTI rate of more than $30 \%$ in this subgroup. Our findings showed similar results with decreased protocol success after low anterior rectal resection; however, our UTI rate was lower (3.7\%), thus promoting a short delay (POD 3) before urinary catheter removal. No anastomosis and increased operative time were associated with increased protocol failure; these criteria probably reflect more advanced tumors with nerve sacrifice and/or frailer patients.
One randomized study that investigated early removal (POD 1) of the urinary catheter within an ERAS program for pelvic colorectal surgery [15] reported interesting results. This policy was combined with the addition of an oral $\alpha$-antagonist. However, the patients were mostly young, with inflammatory bowel disease, low rates of TME (23.9\%), and preoperative CRT (7\%). Therefore, this study does not allow the determination of the optimal duration of postoperative urinary catheterization in patients with rectal cancer. Several nonrandomized studies have evaluated early removal of the urinary catheter within an ERAS program [16-18], but the data were mixed because they included rectal and colonic resec- 
Table 3. Univariate analysis of clinicopathological factors associated with urinary protocol failure

\begin{tabular}{|c|c|c|c|c|}
\hline Variable & Total & Failure & OR $(95 \% \mathrm{Cl})$ & P-value ${ }^{a}$ \\
\hline \multicolumn{5}{|l|}{ Age (yr) } \\
\hline$\geq 70$ & 53 & 7 & $1.4(0.4-4.77)$ & 0.580 \\
\hline$<70$ & 82 & 8 & Reference & \\
\hline \multicolumn{5}{|l|}{ Sex } \\
\hline Male & 86 & 12 & $0.4(0.11-1.5)$ & 0.270 \\
\hline Female & 49 & 3 & Reference & \\
\hline \multicolumn{5}{|l|}{ Body mass index $\left(\mathrm{kg} / \mathrm{m}^{2}\right)$} \\
\hline$\geq 30$ & 22 & 7 & $0.16(0.05-0.51)$ & 0.003 \\
\hline$<30$ & 113 & 8 & Reference & \\
\hline \multicolumn{5}{|l|}{ ASA PS classification } \\
\hline III-IV & 20 & 5 & $0.28(0.08-0.95)$ & 0.048 \\
\hline$|-| \mid$ & 115 & 10 & Reference & \\
\hline \multicolumn{5}{|l|}{ Urology history } \\
\hline Yes & 24 & 4 & $0.55(0.16-1.9)$ & 0.550 \\
\hline No & 111 & 11 & Reference & \\
\hline \multicolumn{5}{|l|}{ Diabetes mellitus } \\
\hline Yes & 15 & 3 & $2.23(0.35-4.77)$ & 0.370 \\
\hline No & 120 & 12 & Reference & \\
\hline \multicolumn{5}{|l|}{ Antiaggregant platelet } \\
\hline Yes & 22 & 8 & $0.12(0.04-0.37)$ & $<0.001$ \\
\hline No & 111 & 7 & Reference & \\
\hline \multicolumn{5}{|l|}{ Prior radiotherapy } \\
\hline Yes & 78 & 12 & $0.3(0.08-1.12)$ & 0.110 \\
\hline No & 77 & 3 & Reference & \\
\hline \multicolumn{5}{|l|}{ Operative time (min) } \\
\hline$>326$ & 65 & 12 & $0.21(0.06-0.8)$ & 0.020 \\
\hline$\leq 326$ & 70 & 3 & Reference & \\
\hline \multicolumn{5}{|l|}{ Surgical procedure } \\
\hline PME & 43 & 0 & - & 0.030 \\
\hline TME & 77 & 15 & & \\
\hline \multicolumn{5}{|l|}{ Anastomosis } \\
\hline No (Hartman procedure) & 11 & 5 & $0.1(0.03-0.41)$ & 0.003 \\
\hline Yes & 124 & 10 & Reference & \\
\hline \multicolumn{5}{|l|}{ Balanced IV fluid therapy } \\
\hline Yes & 116 & 8 & $7.87(2.43-25.5)$ & 0.001 \\
\hline No & 19 & 7 & Reference & \\
\hline \multicolumn{5}{|l|}{ Predictable CRM (mm) } \\
\hline$\geq 1$ & 72 & 5 & $0.39(0.13-1.23)$ & 0.170 \\
\hline$<1$ & 63 & 10 & Reference & \\
\hline \multicolumn{5}{|l|}{ ERAS compliance } \\
\hline Complete (20 items/20) & 67 & 2 & 7.68 (1.66-35.52) & 0.007 \\
\hline Not complete & 68 & 13 & Reference & \\
\hline \multicolumn{5}{|l|}{ Opioid administration } \\
\hline Yes & 62 & 9 & $0.52(0.15-1.81)$ & 0.290 \\
\hline No & 73 & 6 & Reference & \\
\hline \multicolumn{5}{|l|}{ Postoperative NSAID use } \\
\hline Yes & 121 & 7 & $21.7(5.89-80.07)$ & $<0.001$ \\
\hline No & 14 & 8 & Reference & \\
\hline
\end{tabular}

(Continued to the next)
Table 3. Continued

\begin{tabular}{lrccc}
\hline Variable & Total & Failure & OR $(95 \% \mathrm{Cl})$ & P-value $^{\mathrm{a}}$ \\
\hline Early mobilization & & & & \\
No & 22 & 8 & $0.12(0.01-1.99)$ & 0.210 \\
Yes & 133 & 7 & Reference & \\
\hline
\end{tabular}

$\mathrm{OR}$, odds ratio; $\mathrm{Cl}$, confidence interval; $\mathrm{ASA}$, American Society of Anesthesiologists; PS, physical status; PME, partial mesorectal excision; TME, total mesorectal excision; IV, intravenous; CRM, circumferential radial margin; ERAS, Enhanced Recovery After Surgery; NSAID, nonsteroidal anti-inflammatory drugs.

${ }^{a}$ Chi-square, Fisher, or Wilcoxon test.

tions with or without epidural analgesia; however, this technique is no longer recommended for laparoscopic colorectal resection $[5,19]$. Furthermore, the patients were highly selected; those with a history of urinary disease were excluded.

In the rectal resection subgroup, AUR rates from 19.2\% to $33 \%$ have been reported after catheter removal on POD 2 or POD 3. Overall compliance with the ERAS program was unclear in these studies; consequently, no unequivocal conclusions could be drawn.

Our study is the first to establish a link between optimal compliance with an ERAS program and urinary outcomes after rectal cancer surgery. Some well-known risk factors for AUR and UTI are not modifiable, such as age, male sex, BMI, pre-existing urologic symptoms, and diabetes mellitus [20-22]. Interestingly, a history of urinary disease was not associated with an increased rate of protocol failure despite the absence of preoperative urodynamic evaluations in our protocol. Only obesity, ASA PS classification III, and platelet antiaggregant medication were associated with an increased rate of protocol failure. In addition, optimal compliance and certain ERAS measures seem to have greater influences on urinary outcomes.

As already suggested for the prevention of postoperative ileus [23], it seems that implementation of an ERAS program with optimal compliance is the key to faster recovery and, in our study, to better urinary outcomes. ERAS programs aim to counteract inflammatory responses by favoring a minimally invasive approach, early ambulation, avoidance of opioid administration, and balanced fluid management. Obtaining optimal compliance with the ERAS for rectal surgery is an indisputable new challenge for the entire surgical team. In our study, opioid administration was not associated with protocol failure, but we did not investigate a possible dose-dependent effect.

Conversely, NSAID administration was associated with success in our study, thus confirming the proven ability of NSAID to reduce pain and inflammation and their morphine-sparing effects. Other measures contributing to reduced opioid use were ultrasound-guided transversus abdominis plane blocks [24] and systemic lidocaine [25]. Finally, $8 \mathrm{mg}$ of dexamethasone just before the start of the procedure has been proven to reduce AUR after laparoscopic inguinal hernia repair [26]; therefore, its possible 
benefit for colorectal surgery should be investigated.

Our study had some limitations. First, there was a lack of objective documentation of preoperative urodynamic dysfunction, especially after CRT. Second, our AUR definition was not based on radiological criteria and our UTI definition was not based on a systematic bacteriological analysis. We do not perform these examinations routinely; however, we wanted to evaluate a protocol that would reflect daily practice and could be applied to all patients undergoing rectal cancer resection without exclusion criteria. Moreover, the prospective evaluations, the number of unselected patients included during a short period, and high compliance with the ERAS program allowed us to propose a relevant discussion. Urinary catheterization with oral alpha-blockers for 48 hours after minimally invasive TME integrated into an ERAS protocol appears to be a well-balanced strategy for avoiding the risk of AUR and limiting the UTI risk as well as allowing early ambulation. Therefore, it is one of the key factors for the success of ERAS.

In conclusion, withdrawal of the urinary catheter on POD 3 after laparoscopic rectal surgery was successfully achieved in nearly $90 \%$ of patients and should be safely implemented within the ERAS program. For patients with risk factor for failure other than antiaggregant platelet medication (i.e., obesity, ASA PS classification > II, no anastomosis, and prolonged procedure), a suprapubic catheter should be considered as an alternative.

\section{CONFLICT OF INTEREST}

No potential conflict of interest relevant to this article was reported.

\section{ACKNOWLEDGMENTS}

We thank Pr. P. Viens and Dr. J.L. Blache for their contributions to our program implementation, and Pr. E. Lambaudie for providing logistical support to our Institutional Mini-Invasive Surgery Program (DIMI).

\section{REFERENCES}

1. Ratnaval CD, Renwick P, Farouk R, Monson JR, Lee PW. Suprapubic versus transurethral catheterisation of males undergoing pelvic colorectal surgery. Int J Colorectal Dis 1996;11:177-9.

2. Garibaldi RA, Burke JP, Dickman ML, Smith CB. Factors predisposing to bacteriuria during indwelling urethral catheterization. N Engl J Med 1974;291:215-9.

3. Benoist S, Panis Y, Denet C, Mauvais F, Mariani P, Valleur P. Optimal duration of urinary drainage after rectal resection: a randomized controlled trial. Surgery 1999;125:135-41.

4. Stahl TJ, Gregorcyk SG, Hyman NH, Buie WD; Standards Practice Task Force of The American Society of Colon and Rectal Surgeons. Practice parameters for the prevention of venous thrombosis. Dis Colon Rectum 2006;49:1477-83.
5. Nygren J, Thacker J, Carli F, Fearon KC, Norderval S, Lobo DN, et al. Guidelines for perioperative care in elective rectal/pelvic surgery: Enhanced Recovery After Surgery (ERAS ${ }^{\circledR}$ ) Society recommendations. Clin Nutr 2012;31:801-16.

6. Tammela T, Kontturi M, Lukkarinen O. Postoperative urinary retention. I. Incidence and predisposing factors. Scand J Urol Nephrol 1986;20:197-201.

7. Kidd EA, Stewart F, Kassis NC, Hom E, Omar MI. Urethral (indwelling or intermittent) or suprapubic routes for short-term catheterisation in hospitalised adults. Cochrane Database Syst Rev 2015; (12):CD004203.

8. Hojo K, Vernava AM 3rd, Sugihara K, Katumata K. Preservation of urine voiding and sexual function after rectal cancer surgery. Dis Colon Rectum 1991;34:532-9.

9. Kneist W, Heintz A, Junginger T. Major urinary dysfunction after mesorectal excision for rectal carcinoma. Br J Surg 2005;92:230-4.

10. MacFarlane JK, Ryall RD, Heald RJ. Mesorectal excision for rectal cancer. Lancet 1993;341:457-60.

11. Kang SB, Park JW, Jeong SY, Nam BH, Choi HS, Kim DW, et al. Open versus laparoscopic surgery for mid or low rectal cancer after neoadjuvant chemoradiotherapy (COREAN trial): short-term outcomes of an open-label randomised controlled trial. Lancet Oncol 2010;11:637-45.

12. Lee SY, Kang SB, Kim DW, Oh HK, Ihn MH. Risk factors and preventive measures for acute urinary retention after rectal cancer surgery. World J Surg 2015;39:275-82.

13. Kwaan MR, Lee JT, Rothenberger DA, Melton GB, Madoff RD. Early removal of urinary catheters after rectal surgery is associated with increased urinary retention. Dis Colon Rectum 2015;58:4015.

14. Zmora O, Madbouly K, Tulchinsky H, Hussein A, Khaikin M. Urinary bladder catheter drainage following pelvic surgery: is it necessary for that long? Dis Colon Rectum 2010;53:321-6.

15. Patel DN, Felder SI, Luu M, Daskivich TJ, N Zaghiyan K, Fleshner P. Early urinary catheter removal following pelvic colorectal surgery: a prospective, randomized, noninferiority trial. Dis Colon Rectum 2018;61:1180-6.

16. Sarin A, Litonius ES, Naidu R, Yost CS, Varma MG, Chen LL. Successful implementation of an Enhanced Recovery After Surgery program shortens length of stay and improves postoperative pain, and bowel and bladder function after colorectal surgery. BMC Anesthesiol 2016;16:55.

17. Stubbs BM, Badcock KJ, Hyams C, Rizal FE, Warren S, Francis D. A prospective study of early removal of the urethral catheter after colorectal surgery in patients having epidural analgesia as part of the Enhanced Recovery After Surgery programme. Colorectal Dis 2013;15:733-6.

18. Ghuman A, Kasteel N, Karimuddin AA, Brown CJ, Raval MJ, Phang PT. Urinary retention in early urinary catheter removal after colorectal surgery. Am J Surg 2018;215:949-52.

19. Hübner M, Blanc C, Roulin D, Winiker M, Gander S, Demartines N. Randomized clinical trial on epidural versus patient-controlled 
analgesia for laparoscopic colorectal surgery within an enhanced recovery pathway. Ann Surg 2015;261:648-53.

20. Kang CY, Chaudhry OO, Halabi WJ, Nguyen V, Carmichael JC, Mills S, et al. Risk factors for postoperative urinary tract infection and urinary retention in patients undergoing surgery for colorectal cancer. Am Surg 2012;78:1100-4.

21. Kin C, Rhoads KF, Jalali M, Shelton AA, Welton ML. Predictors of postoperative urinary retention after colorectal surgery. Dis Colon Rectum 2013;56:738-46.

22. Wu AK, Auerbach AD, Aaronson DS. National incidence and outcomes of postoperative urinary retention in the Surgical Care Improvement Project. Am J Surg 2012;204:167-71.

23. Grass F, Slieker J, Frauche P, Solà J, Blanc C, Demartines N, et al.
Postoperative urinary retention in colorectal surgery within an enhanced recovery pathway. J Surg Res 2017;207:70-6.

24. Baeriswyl M, Kirkham KR, Kern C, Albrecht E. The analgesic efficacy of ultrasound-guided transversus abdominis plane block in adult patients: a meta-analysis. Anesth Analg 2015;121:1640-54.

25. De Oliveira GS Jr, Fitzgerald P, Streicher LF, Marcus RJ, McCarthy RJ. Systemic lidocaine to improve postoperative quality of recovery after ambulatory laparoscopic surgery. Anesth Analg 2012; 115:262-7.

26. Denham M, Donovan K, Wetoska N, Kuchta K, Carbray J, Linn JG, et al. Effects of dexamethasone on postoperative urinary retention after laparoscopic inguinal hernia repair. Surg Endosc 2019; 33:3008-13. 